\title{
Sur la situation intenable des psychothérapeutes (h/F) en Suisse
}

\section{Déclaration sur l'article de Peter Schulthess : "Allemagne : Nouvelle loi sur la psychothérapie avant l'obtention du diplôme » (à jour !1/2019)}

\section{Kurt Roth}

L'Allemagne nous montre la voie : Enfin, une réglementation raisonnable, juste et orientée vers l'avenir de la psychothérapie sera mise en place, létude (cursus/filière) directe deviendra possible. La psychothérapie ne devrait plus être un appendice d'aucune science (essentiellement la psychologie, la médecine), mais devrait devenir une discipline scientifique indépendante.

En revanche, la situation en Suisse semble désastreuse. Les trois associations professionnelles FSP, ASP et SBAP ont terriblement échoué. Depuis trop longtemps, les associations ne se sont qu'interrogées sur les sciences qui constituent la base de la psychothérapie et sur les études qui devraient permettre d'accéder au titre de formation postgrade " psychothérapeute $(\mathrm{h} / \mathrm{f})$ ». Les conséquences de cet échec : La psychothérapie n'est pas une science indépendante qui fait lobjet de recherches et qui est enseignée dans les universités. Aujourd'hui, en Suisse, seul un master en psychologie permet de poursuivre une formation postgrade de psychothérapeute (h/f) "psychologique»(dont $80 \%$ de femmes). En tant que directrice d'une clinique de toxicomanie, j’ai pu accompagner de nombreux psychologues (femmes) dans leurs premiers pas dans leur profession après leurs études. Après avoir obtenu une maîtrise en psychologie à lâge de 26 ans et choisi une carrière de psychothérapeute, elles ne peuvent pas se lancer et devenir actives sur le plan professionnel. Comme disent les professeurs de psychologie des universités : l'étude de la psychologie n'est pas seulement une formation professionnelle, mais une formation scientifique. Si une psychologue souhaite devenir psychothérapeute après ses études, elle peut acquérir le titre de formation postgrade à ses propres frais. Elle doit travailler - souvent dans des conditions précaires - pendant un ou deux ans comme assistante psychologue dans une clinique psychiatrique. Bien sûr, il y a de telles cliniques ici et ailleurs. Mais certains profitent de la situation sans vergogne et paient de très mauvais salaires parce que la demande pour ces emplois est élevée. En outre, elle doit suivre une formation postgrade de psychothérapeute dans un institut de formation agréé. Le coût sélève à 50000 à 60000 francs - sans tenir compte de la perte de revenus, car 100 pour cent du travail ne peut être effectué en plus de la formation postgrade. Si l'on tient compte de la perte de $20 \%$ de l'emploi, les coûts sélèveraient à au moins 100000 francs pour une période de formation postgrade de quatre ans. En règle générale, le psychologue doit financer ces coûts complètement ou en grande partie de sa poche. Pour comparaison : les cliniques reçoivent des contributions de l'État pour l'emploi de médecins assistants. Les futurs spécialistes bénéficient donc de bonnes conditions d'emploi et de formation postgrade à l'issue de leur master et de leur examen d'État.

Vers lâge de 30 ans, lorsque tout s'est bien passé, la psychologue est finalement devenue une psychothérapeute reconnue par le gouvernement fédéral. Cet important effort financier et en temps devrait au moins se traduire par un bon salaire et de bonnes conditions de travail. Ça n’a pas l'air d'être très rose là-bas non plus. C'est la même chose pour toutes les professions d'assistance - et aujourd'hui encore, la plupart du temps, ce sont les femmes qui les exercent : Le grand idéalisme qui sous-tend ces activités est souvent exploité socialement et mal rémunéré financièrement. Aux frais de l'assurance des soins obligatoire (OKP), la psychothérapeute ne peut travailler qu'en tant qu’employée d’un médecin, dit " délégué ». Aujourd'hui, le principe de lordre doit venir remplacer le principe de délégation. Une petite amélioration quand un tel cas se produit. Cependant, je suis très enthousiaste au sujet de l'indemnisation, qui est prévue pour 60 minutes de psychothérapie prescrite par un médecin. De plus, les charges administratives et réglementaires ne faciliteront pas exactement le travail.

À mon avis, les associations professionnelles de psychothérapie en Suisse ont un grand besoin d'action en termes de politique professionnelle. Lobjectif doit être de faire de la psychothérapie une discipline scientifique indépendante dans les universités et de permettre des études (cursus/filières) directes qui, après une période d'assistance clinique, autorisent une pratique professionnelle indépendante et une facturation indépendante via l'OKP (voir Allemagne). La formation et la

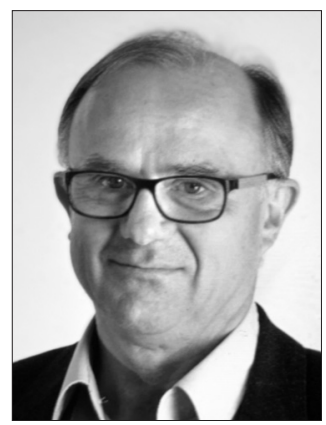


formation postgrade en tant que psychothérapeute (femme) en Suisse, financées et organisées exclusivement par le secteur privé, est un scandale et une exploitation éhontée des psychothérapeutes (femmes) potentielles par les législateurs et le secteur public.

PS : Juste une petite comparaison : Devenez informaticien (95\% d'hommes) ou bien informaticienne. On peut déjà être informaticienne après un apprentissage de trois ans, puis se former par l'intermédiaire des hautes écoles spécialisées. Aujourd'hui, un informaticien facture entre 200 et
250 francs suisses de l'heure - et pas seulement dans le système de santé. De plus, il y a des factures de temps et de kilométrage s'il doit travailler directement sur place. De plus, il peut déjà gagner beaucoup d'argent en tant que jeune professionnel.

Kurt Roth est psychothérapeute reconnu au niveau fédéral, directeur de la clinique de traitement des dépendances d'Egliswil et directeur général de la Fondation pour la thérapie sociale (retraité depuis le 1er août 2019). Il a été membre du comité de l'ASP de 2006 à 2013.

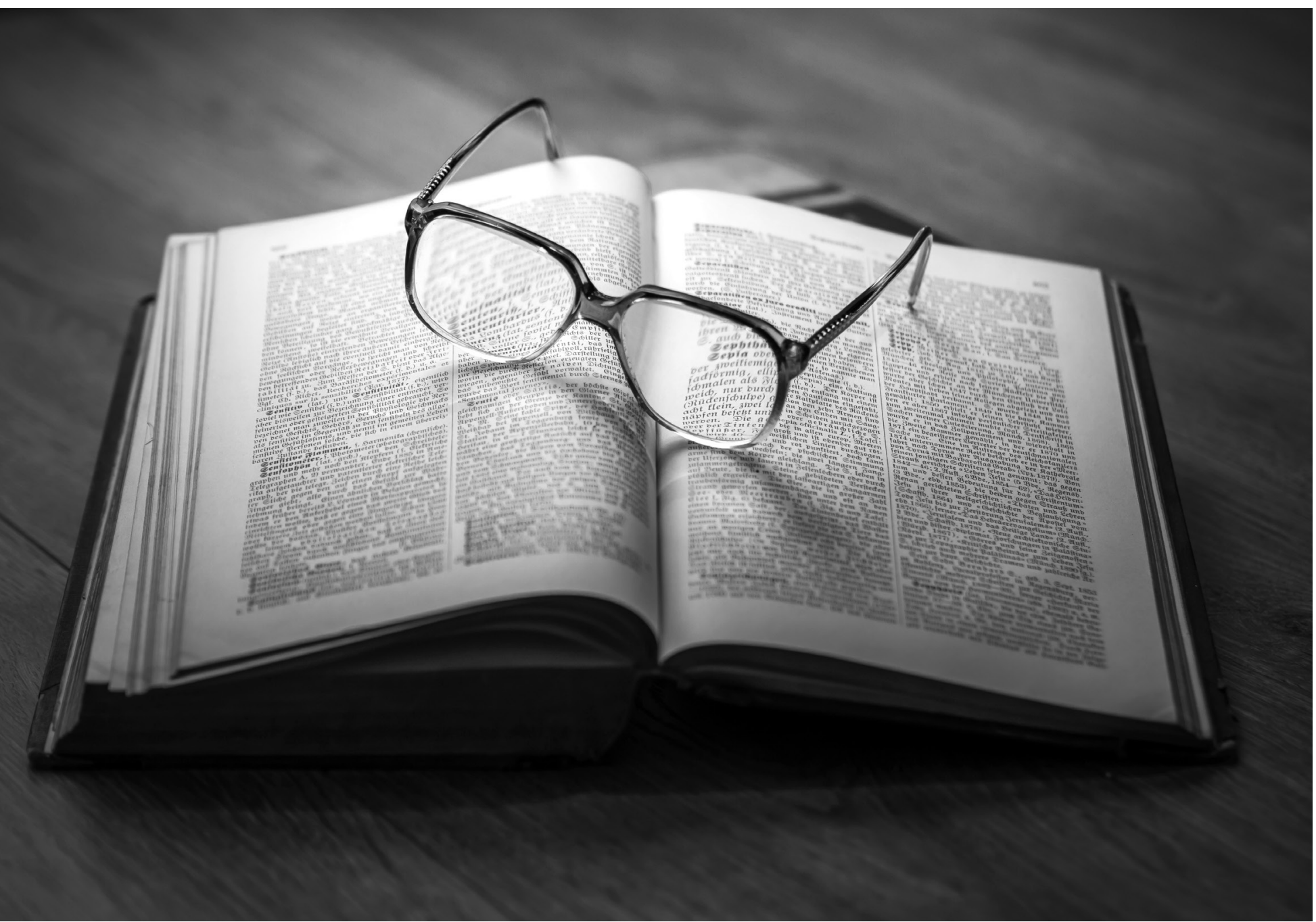

